\title{
Homens acometidos de câncer de próstata e suas vulnerabilidades
}

\author{
Men with prostate cancer and their vulnerabilities \\ Hombres con cáncer de próstata y sus vulnerabilidades
}

Elizabeth Rose Costa Martins ORCID: https://orcid.org/0000-0001-5947-5535 Universidade do Estado do Rio de Janeiro, Brasil E-mail: oigresrose@gmail.com

Nizélia Ferreira da Silva Floro Rosa ORCID: https://orcid.org/0000-0003-2841-0848 Universidade do Estado do Rio de Janeiro, Brasil E-mail: nizelia.floro@gmail.com

Karoline Lacerda de Oliveira ORCID: https://orcid.org/0000-0001-8755-5858 Universidade do Estado do Rio de Janeiro, Brasil E-mail: lacerdakarol@hotmail.com

Andressa da Silva Medeiros ORCID: https://orcid.org/0000-0001-8677-2543 Universidade do Estado do Rio de Janeiro, Brasil E-mail: andressamedeirosmedeiros2@gmail.com Jady Assis de Souza

ORCID: https://orcid.org/0000-0003-2918-9209 Universidade do Estado do Rio de Janeiro, Brasil E-mail: jadysouza@hotmail.com

Letícia Guimarães Fassarella ORCID: https://orcid.org/0000-0002-3903-7383 Universidade do Estado do Rio de Janeiro, Brasil E-mail: lelefassarella@gmail.com

Cristiane Maria Amorim Costa ORCID: https:// orcid.org/0000-0003-1089-2092 Universidade do Estado do Rio de Janeiro, Brasil E-mail: cmacosta64@gmail.com

Priscila Marques Ranquine ORCID: https://orcid.org/0000-0002-6580-6203 Universidade do Estado do Rio de Janeiro, Brasil E-mail:priscilaranquine@hotmail.com

Nathalia Figueiredo de Araújo ORCID: https://orcid.org/0000-0002-4645-3932 Universidade do Estado do Rio de Janeiro, Brasil E-mail:nathaliafigueiredo17@gmail.com

Isabele Souza de Moraes Rego ORCID: https;//orcid.org/0000-0002-7324-0449 Universidade do Estado do Rio de Janeiro, Brasil. E-mail:isabele.smr@gmail.com Beatriz da Costa Pereira ORCID: https://orcid.org/0000-0002-0675-8053 Universidade do Estado do Rio de Janeiro, Brasil E-mail:beacosta008@gmail.com

Fabiana Cristina Silva da Rocha ORCID: https://orcid.org/0000-0001-7665-0550 Universidade do Estado do Rio de Janeiro, Brasil E-mail: fabianacsrocha@hotmail.com

\begin{abstract}
Resumo
Objetivos: Identificar as práticas adotadas pelo homem para prevenção de câncer de próstata; descrever as condutas de saúde de homens acometidos com câncer de próstata e discutir seu impacto na vida do homem. Metodologia: Estudo descritivo e exploratório, de natureza qualitativa, desenvolvido com 25 homens a partir de 55 anos, com diagnóstico de câncer de próstata na unidade urológica de um hospital universitário situado no município do Rio de Janeiro. O instrumento de coleta de dados foi a entrevista semiestruturada e, para análise dos dados, foi aplicada a técnica de análise de conteúdo, conforme as seguintes etapas: pré-análise, exploração do material e tratamento dos resultados, inferência e interpretação. Resultados e Discussão: O estudo evidenciou que mesmo entendendo a necessidade de cuidar da sua saúde, através da prevenção de doenças, só buscaram atendimento quando os sintomas começaram a
\end{abstract}


incomodar de alguma forma. Isto reforça não só o déficit no autocuidado, como também a dificuldade de se perceber vulnerável e responsável pela sua saúde. Além disso, muitos são os sentimentos, expectativas e significados atribuídos pelos homens após o diagnóstico, principalmente os que envolvem a prática sexual. Considerações finais: É imprescindível um acolhimento em sua integralidade na perspectiva de gênero, trazendo, assim, um desafio de se pensar a saúde do homem voltada à promoção da saúde e à prevenção de doenças, instituindo a educação em saúde como um pilar para fortalecer o vínculo da população masculina com os serviços de saúde.

Palavras-chave: Saúde do homem; Câncer de próstata; Vulnerabilidade; Prevenção; Enfermagem.

\begin{abstract}
Objectives: Identify the practices adopted by men to prevent prostate cancer; describe the health behaviors of men affected with prostate cancer and discuss their impact on men's lives. Methodology: Descriptive and exploratory study, with a qualitative approach, developed with 25 men aged 55 years and over, diagnosed with prostate cancer in the urological unit of a university hospital located in the city of Rio de Janeiro. The data collection instrument was the semi-structured interview and, for data analysis, the content analysis technique was applied, according to the following steps: pre-analysis, material exploration and treatment of results, inference and interpretation. Results and Discussion: The study showed that even understanding the need to take care of their health, through disease prevention, they only sought care when symptoms began to bother them in some way. This reinforces not only the deficit in self-care, but also the difficulty of perceiving oneself as vulnerable and responsible for one's health. In addition, there are many feelings, expectations and meanings attributed by men after diagnosis, especially those involving sexual practice. Final considerations: It is essential to embrace it in its entirety in the gender perspective, thus bringing a challenge to think about men's health aimed at promoting health and preventing diseases, establishing health education as a pillar to strengthen the link of the male population with health services.
\end{abstract}

Keywords: Men's health; Prostate cancer; Vulnerability; Prevention; Nursing.

\begin{abstract}
Resumen
Objetivos: Identificar las prácticas adoptadas por los hombres para prevenir el cáncer de próstata; describir los comportamientos de salud de los hombres afectados por el cáncer de próstata y discutir su impacto en la vida de los hombres. Metodología: Estudio descriptivo y exploratorio, carácter cualitativo, desarrollado con 25 hombres de 55 años y más, diagnosticados de cáncer de próstata en la unidad de urología de un hospital universitario ubicado en la ciudad de Río de Janeiro. El instrumento de recolección de datos fue la entrevista semiestructurada y, para el análisis de datos, se aplicó la técnica de análisis de contenido, de acuerdo con los siguientes pasos: preanálisis, exploración de materiales y tratamiento de resultados, inferencia e interpretación. Resultados y Discusión: El estudio mostró que aun entendiendo la necesidad de cuidar su salud, a través de la prevención de enfermedades, solo buscaban atención cuando los síntomas comenzaban a molestarlos de alguna manera. Esto refuerza no solo el déficit en el autocuidado, sino también la dificultad de percibirse como vulnerable y responsable de la propia salud. Además, son muchos los sentimientos, expectativas y significados atribuidos por los hombres tras el diagnóstico, especialmente los que involucran la práctica sexual. Consideraciones finales: Es fundamental acogerlo en su totalidad en la perspectiva de género, planteando así un desafío para pensar en la salud del hombre orientado a promover la salud y prevenir enfermedades, estableciendo la educación para la salud como un pilar para fortalecer el vínculo de la población masculina con la salud. servicios.
\end{abstract}

Palabras clave: Salud del hombre; Cáncer de próstata; Vulnerabilidad; Prevención; Enfermería.

\title{
1. Introdução
}

O câncer (CA) de próstata é, ainda hoje, um problema de saúde pública no Brasil, sendo um dos cânceres que mais levam homens a óbito no país e, além disso, o segundo de maior incidência. A doença tem uma evolução lenta, porém seus sinais e sintomas são inexistentes em grande parte das vezes nos estágios iniciais, tornando ainda mais difícil a detecção da doença quando o indivíduo acometido não realiza consultas preventivas (Lyra et al., 2020).

$\mathrm{O}$ aumento da incidência dos tipos de patologias encontradas em homens como o CA prostático e a hiperplasia prostática benigna (HPB) deve-se a diversos fatores, tais como o estilo de vida, herança genética e idade, visto que comprovadamente acomete homens entre 55 e 70 anos (Silva et al., 2018).

A próstata é uma glândula exclusivamente presente em homens, sendo responsável pela produção de um líquido que compõe parte do sêmen, o qual nutre e dá proteção aos espermatozoides liberados na ejaculação. Está localizada anteriormente ao reto e abaixo da bexiga, tendo também íntima relação com parte da uretra masculina (Brasil, 2014). 
Ao longo de toda a vida, as células que compõem os tecidos do organismo são renovadas; no entanto, fatores externos, como a alimentação inadequada ou com aporte nutricional hipossuficiente, sedentarismo, obesidade, tabagismo, etilismo, entre outros, podem favorecer o desarranjo celular e contribuir para a proliferação exacerbada, levando à formação de tumores malignos (CA) ou benignos (HPB) (Brasil, 2014).

Os fatores genéticos são ainda mais influentes quando há herança genética de CA de próstata ou HBP em familiares de primeiro grau, sendo indivíduos negros ou pardos os com maior predisposição para o desenvolvimento da doença (Silva et al., 2018).

Segundo dados do último censo realizado pelo Instituto Brasileiro de Geografia e Estatística (IBGE) no ano de 2010, existiam em território brasileiro em torno de 17.893.451 milhões de homens, entre a faixa etária de 50 e 80 anos ou mais. Deste total, a maior parte está localizada na Região Sudeste, com aproximadamente 8.205.826 milhões de homens (IBGE, 2010).

O aumento da expectativa de vida em países desenvolvidos e em desenvolvimento ou emergentes, como o Brasil, leva ao questionamento e ao conhecimento da crescente taxa de incidência de CA prostático e HPB, visto que ambas são patologias intimamente relacionadas ao envelhecimento. Estudos mostram que a HPB afeta 50\% dos homens acima dos 55 anos de idade e 90\% dos indivíduos após a oitava década de vida (Silva et al., 2018).

A falta de cuidado do homem está presente de forma intrínseca na sociedade até o presente momento, pois ainda há uma construção patriarcal que interpreta o homem como um ser que não é acometido por doenças, um ser forte, que não pode apresentar fraquezas. Essa crença vai de encontro ao ideário feminino, marcado pela necessidade de cuidado; deste modo, perpetua-se o cuidado como uma atribuição apenas da mulher, o que distancia cada vez mais o homem das suas reais necessidades de saúde e, consequentemente, o colocando em posição mais vulnerável (Martins et al., 2020a).

$\mathrm{O}$ conceito de vulnerabilidade masculina, quando abordado na saúde, remete à fragilidade e à propensão por parte dos homens em adquirirem patologias. Ou seja, o público masculino apresenta maiores chances de desenvolver doenças devido à ausência, durante toda a sua vida, da prática do cuidado (Martins et al., 2020b).

Sabe-se também que, além do estereótipo de força atribuída ao homem, a falta de conhecimento sobre seu próprio corpo e sua saúde é outro fator que se soma à ausência de cuidados por parte desse público. Do mesmo modo, a jornada de trabalho de muitos contribui de maneira negativa para o acesso aos centros de saúde de forma preventiva, uma vez que geralmente são eles os principais provedores de renda de seu lar (Martins et al., 2020c).

Acredita-se que a masculinidade imposta aos homens desde o seu nascimento seja um dos elementos predominantes na dificuldade de implementação de políticas de prevenção e até mesmo no tratamento de comorbidades, visto a escassez de procura ao atendimento em saúde, especialmente por homens adultos e idosos.

Apesar de a população masculina ter o conhecimento sobre o câncer de próstata, ainda existe muita resistência para a adesão às consultas e exames, por haver uma masculinidade como produto de interações sociais entre homens, gerando medo e constrangimento que os impedem de participar de etapas fundamentais para a detecção precoce, como o caso do exame de toque retal, que é o mais rejeitado por eles (Lyra et al., 2020).

O medo e o preconceito impostos socialmente sobre o homem e sobre o próprio exame de diagnóstico para a doença são fatores que dificultam o diagnóstico precoce e a adesão ao tratamento (Brasil, 2015). O exame de toque retal é ainda o meio mais fidedigno de obtenção do diagnóstico de aumento do tamanho da próstata, que pode ou não ser diagnosticado como câncer. Com isso, é de suma importância mostrar essa realidade para a população masculina para que ela se conscientize e desmistifique o medo sobre o exame para diagnóstico e para a doença (Martins et al., 2020a).

A necessidade de acesso e manutenção da população masculina nas unidades básicas de saúde tornou-se um desafio para os profissionais, diante da resistência do homem em cuidar da sua saúde. Tal fato torna o papel da enfermagem ainda 
mais fundamental para conscientizar e atrair essa população, de maneira que cada grupo seja interpretado individualmente e esclarecido sobre a doença, de acordo com suas singularidades (Lyra et al., 2020).

Vale ressaltar que os homens, quando buscam atendimento, já se encontram em estágio intermediário para avançado da doença, com escore de Gleason 7/6, evoluindo para 8 (Mota \& Barros, 2018). Nessas circunstâncias, a recuperação é dificultada, pois pode atingir outras estruturas do sistema reprodutor masculino e das cadeias linfonodais pélvicas, diferentemente da situação em que a detecção é precoce e localizada, aumentando a expectativa de vida exponencialmente (Araújo, Conceição, Oliveira \& Zago, 2015).

A Política Nacional de Atenção Integral à Saúde do Homem (PNAISH) foi criada para ajudar no acesso aos serviços de saúde à população do sexo masculino; entretanto, a maior proporção desses indivíduos com neoplasia prostática nunca ouviu falar desta política. Nesse sentido, evidencia-se, mais uma vez, a necessidade de informações adequadas para estes pacientes e para o restante da população, através de um maior engajamento entre os profissionais, o sistema de saúde e os pacientes (Rodrigues, Sá \& Zangrande, 2016).

A informação é um importante aliado à promoção e prevenção à saúde da população masculina, sendo necessária para a adesão dos homens ao tratamento, mostrando como a detecção precoce é fundamental para a expectativa e qualidade de vida. Cabe, então, aos profissionais de saúde fornecerem essas informações para conscientização da população masculina, com o planejamento e implementação de estratégias que visam facilitar a compreensão das informações (Rodrigues, Sá \& Zangrande, 2016).

Além de informação, pacientes em tratamento oncológico de próstata necessitam de um cuidado e planejamento personalizados, levando sempre em consideração a individualidade de cada doente, como o estadiamento da neoplasia, idade, existência de doenças crônicas, saúde mental, recursos disponíveis na unidade de atendimento, bem como a opinião do paciente diante das condutas terapêuticas que serão realizadas, proporcionando-lhe maior autonomia (Mota \& Barros, 2018).

O apoio financeiro também é de suma importância, uma vez que essa população tem seu desempenho laboral prejudicado, sendo necessária até a sua ausência para melhor adesão ao tratamento, o que interfere diretamente na renda desses trabalhadores. Com isso, existem leis que dão suporte para que todos os pacientes possam usufruir dos seus direitos e benefícios, como o auxílio-doença, oferecido pelo governo (Rodrigues, Sá \& Zangrande, 2016).

Muitos são os impactos que o câncer prostático pode produzir; porém, inicialmente, a doença não produz sinais e sintomas suficientes, mas com o avançar da doença neoplásica, pode-se identificar alterações no funcionamento fisiológico que interferem na qualidade de vida e autoestima dos homens acometidos. Isso mostra alguns dos motivos da dificuldade por parte dos pacientes oncológicos em seguir o tratamento - e até mesmo na detecção precoce do câncer de próstata, interferindo no prognóstico (Rego et al., 2020).

Com este estudo, pretende-se desmistificar o câncer de próstata e possibilitar a consciência da realidade de como os homens se cuidam e o que podem fazer a fim de prevenir o câncer e estimular a valorização da busca pelos serviços de saúde.

Nesse contexto, definiu-se como objeto de estudo homens com câncer de próstata e suas vulnerabilidades. Como objetivos, tem-se: Identificar as práticas adotadas pelo homem para prevenção de câncer de próstata; descrever as condutas de saúde de homens acometidos com câncer de próstata e discutir o impacto do câncer de próstata na vida do homem.

\section{Metodologia}

Estudo descritivo de natureza qualitativa, onde não se preocupa com representatividade numérica, mas com o aprofundamento e compreensão de um grupo social de uma determinada organização. Nela há uma valorização na descrição e explicação dos fenômenos investigados. Além de haver uma preocupação, com as ciências sociais e um nível de realidade que não pode ser quantificado, trabalhando com um universo de significados (Minayo, 2013). 
Realizado em um hospital universitário situado no município do Rio de Janeiro, na enfermaria de urologia, que mantém 10 leitos para a população masculina.

Buscou-se criar alguns critérios para seus participantes, a fim de atender ao objeto de estudo, como: homens a partir de 55 anos, com câncer de próstata, internados no cenário de estudo. O critério de exclusão foi o de homens com hiperplasia prostática benigna. Dessa forma, o estudo contou com 25 participantes.

A pesquisa foi realizada no período de junho a novembro de 2020, após aprovação do Comitê de Ética e Pesquisa sob o n ${ }^{\circ}$ 3.058.467. O estudo seguiu os aspectos éticos e as determinações contidas na Resolução 466/2012 do Conselho Nacional de Saúde.

Como instrumento de coleta de dados, foi utilizada a entrevista semiestruturada, que parte de certos questionamentos básicos apoiados em teorias que interessam à pesquisa e que, em seguida, oferecem amplo campo de interrogativas (Minayo, 2013).

A entrevista foi composta de duas partes: a primeira, caracterizando os participantes segundo a faixa etária, estado civil, escolaridade e ocupação; a segunda, com questões abertas sobre as práticas adotadas pelos homens para prevenir doenças e as condutas de saúde de homens acometidos com câncer de próstata.

Vale ressaltar que foram realizadas 25 entrevistas, conforme a disponibilidade dos homens, de forma individualizada, em um espaço apropriado para a sua realização, sem comprometer seu desenvolvimento.

Cada participante recebeu um termo de consentimento livre e esclarecido (TCLE), no qual todos foram orientados quanto aos objetivos do estudo, a relevância, a metodologia, assim como a garantia da participação voluntária, do sigilo e do anonimato, da possibilidade de desistência da participação na pesquisa e dos possíveis riscos mínimos a ela relacionados.

O número de participantes inseridos no estudo foi apoiado no critério de saturação, cuja base é a percepção do pesquisador em relação à compreensão da lógica dos participantes, do grupo ou da coletividade estudada e a identificação de que esse conhecimento reflete a totalidade das múltiplas dimensões do objeto do estudo em questão (Minayo, 2013).

Com o objetivo de resguardar a identidade dos participantes, foram utilizados códigos de identificação, optando-se pela escolha da letra $\mathrm{H}$, seguida por um número ordinal identificando a ordem das entrevistas realizadas. As entrevistas foram gravadas em formato de áudio, por meio de um aparelho eletrônico, para garantia da fidedignidade das informações coletadas e, posteriormente, transcritas.

Após a coleta, os dados foram transcritos e organizados. Com a intenção de conhecer os significados por trás dos discursos emanados durante as entrevistas, o método utilizado foi o da análise de conteúdo, pois tudo que é dito é susceptível de ser submetido a este tipo de análise (Bardin, 2016).

O objeto da análise de conteúdo é a palavra, aspecto individual da linguagem; seu objetivo são os significados dos vocábulos, tentando compreender os atores e o ambiente onde estão inseridos; é organizada em três etapas: pré-análise; exploração do material e tratamento dos resultados; inferência e interpretação (Bardin, 2016).

Para a sistematização dos achados, realizou-se leitura flutuante, a qual fez emergir 210 unidades de registro, organizada em duas categorias: $\mathrm{O}$ homem e as práticas de prevenção ao câncer de próstata e $\mathrm{O}$ ser homem vivenciando o câncer de próstata.

\section{Resultados e Discussão}

Os participantes do estudo apresentaram a seguinte caracterização: em relação à faixa etária, destaca-se a predominância de 55 a 59 anos, de 18 (60\%) participantes; de 60 a 65 anos, de 4 (16\%) e acima de 66 anos, 3 (12\%) participantes. Quanto ao estado civil, predominância para casados - $20(80 \%)-; 5(20 \%)$ participantes eram viúvos. Quanto à 
escolaridade, predominância do ensino fundamental, com 16 (64\%), ensino médio com 3 (12\%), nível superior, 4 (16\%), e 2 (8\%) com alfabetização. Em relação à ocupação, 100\% dos participantes encontram-se em atividade profissional.

\subsection{O homem e as práticas de prevenção ao câncer de próstata}

A fim de compreender as práticas de prevenção ao câncer de próstata desenvolvidas pelos homens, pode-se observar as seguintes falas:

Eu não procurava médico para prevenir o câncer. (H.2)

Achei que não tinha nada, até que aconteceu. (H.33)

Nunca pensei em prevenção, também não tinha tempo. (H.10)

Eu pensava que se eu não sentia, então eu não tinha nada. (H.12)

No tocante à relação existente entre os homens e o câncer, assim como as práticas para a prevenção do câncer de próstata, muitos desdobramentos das masculinidades permeiam as atitudes, os comportamentos, os sentimentos e as emoções experenciados pelos homens e seus corpos e constructos de ser masculino.

As masculinidades, nesse sentido, estruturam-se com base na organização social em que os sujeitos estão inseridos e são formados, devendo ser compreendida a partir das suas inserções sociais, reproduções e pelo modo de expressão das identidades de gênero entendidas e explicitadas por homens (Lima, Reis \& Demétrio, 2017.)

Martins et al. (2020a) discutem acerca da prevenção à saúde do homem e como esta atitude está atrelada à figura feminina. Isto é confirmado diante das falas:

Minha esposa descobriu quando tivemos relação sexual; ela percebeu um sangue que não era dela e pediu para eu ir fazer exames. (H.6)

Tinha dores de cabeça, atrás da nuca e meu ombro esquerdo às vezes doía, ficava saltando durante a noite. Então minha esposa marcou um exame e eu não fiz o exame de teimoso. Então em 2018, eu estava com sintomas, até um pouco mais e a minha esposa me fez fazer o check up. (H.10)

A centralidade da mulher, como representante dos cuidados para com a população masculina, mantém-se presente até hoje e fica claro nas falas que é a mulher que precisa, de alguma forma, estimular e até mesmo direcionar o homem a se cuidar (Martins et al., 2020b).

Os índices de morbimortalidade masculina ainda permanecem maiores se comparados ao público feminino, caracterizando que homens morrem mais do que mulheres. Sua porta de entrada no serviço de saúde ainda é uma emergência e não a atenção básica, como preconizado pela Rede de Atenção à Saúde. Desta forma, torna-se um desafio muito grande diminuir esses índices, uma vez que estes só buscam quando o agravo já se instalou e não de maneira preventiva (Brasil, 2008).

Mesmo entendendo a necessidade de cuidar da sua saúde, através da prevenção de doenças, os participantes do estudo só buscaram atendimento quando os sintomas começaram a incomodar de alguma forma. Isto reforça não só o déficit no autocuidado, como também a dificuldade de se perceber vulnerável e responsável pela sua saúde.

Não ia ao médico, corria de médico. Achava que fazia tudo direito. Até que comecei a sentir algumas coisas. (H.1)

Procurei atendimento quando saiu sangue na urina, aí percebi que era grave. (H.15) 
Continuo não indo ao médico, só se me sentir mal, aí vou procurar. (H.33)

Um dado importante é o grau de instrução desses pacientes; grande parte deles apresenta baixa escolaridade, o que pode ter como reflexo a dificuldade de percepção do processo saúde-doença, prejudicando o entendimento da importância de exames preventivos, formas de tratamento, vulnerabilidades, gravidade da doença e até mesmo a aceitação e adesão ao tratamento (Araújo, Conceição, Oliveira \& Zago, 2015).

A prevenção que envolve o câncer de próstata se consolida através do exame de toque retal, que também tem sido um exame temido, pois afeta o imaginário masculino, gerando afastamento de inúmeros homens das ações de prevenção, conforme retratam as falas:

Fazer o exame de toque retal nem pensar. (H.26)

Todo mundo goza o homem sobre o toque retal. (H.7)

Sei que é importante o toque retal, mas eu não faço. (H.30)

Quando fui ao médico, pedi para não fazer o toque retal. (H.14)

A dificuldade na realização do exame de toque retal pode estar relacionada à falta de informação e ao desconhecimento dos homens, mas também tem forte associação com os aspectos simbólicos que se encontram ligados ao seu caráter de invasão, tanto do ponto de vista físico como emocional, além do medo da realização do exame (Lima, Reis \& Demétrio, 2017).

Vale ressaltar que problemas estruturais dos serviços de saúde podem dificultar o acesso e o acolhimento de homens em relação à realização de exames que envolvam as ações de prevenção ao câncer de próstata (Lima \& Aguiar, 2020). Além disso, o preconceito pode impactar a saúde do homem, impedindo-o de procurar por profissionais de saúde, por vergonha e medo de perder a masculinidade.

\section{Eu ainda tenho muita vergonha de fazer o exame de toque retal. (H.17)}

Dizem que podemos deixar de ser homem. (H.34)

Os homens passam a revelar tais sentimentos pelo receio de experienciar o desconforto físico, mas também psicológico, de serem submetidos a determinados procedimentos. De alguma forma, reforçam o imaginário de que seu corpo não foi feito para ser tocado.

Esses embates trazem à tona não apenas questões físicas e patológicas dos homens, mas também questões psicossociais, as quais envolvem as construções sociais da masculinidade que, de alguma forma, acabam interferindo de maneira efetiva na promoção à saúde e prevenção de agravos à saúde da população masculina, tornando-os vulneráveis a doenças, como o câncer de próstata.

\subsection{O ser homem vivenciando o câncer de próstata}

Com o diagnóstico de câncer de próstata, muitos serão os sentimentos e significados atribuídos pelos homens e expectativas serão geradas, principalmente quanto à vida sexual após o diagnóstico.

Segundo Ferrão, Berttinelli e Portella (2017), pacientes que realizam tratamento do câncer de próstata se sentem destituídos de sua masculinidade, pois alguns apresentam incapacidade recorrente - ou até persistente - de ereção, trazendo constrangimentos, desconforto e insatisfação diante de seus parceiros sexuais. 
Minha parte sexual está zero, não estou tendo relação sexual, minha mulher está querendo até me largar. (H.3)

A vontade eu até tenho, mas apresento dificuldade na ereção. (H.11).

Cabe ressaltar a importância de tratar da sexualidade masculina no contexto do adoecimento, que é tão carregado de estigmas, pois tem remetido a ideias que estão entrelaçadas à impotência, disfunção erétil, infertilidade e demais agravos que estão relacionados com o funcionamento do corpo (Gomes, 2008).

Nessa perspectiva, a sexualidade não é compreendida como um conjunto de expressões, comportamentos, atitudes, nem como contato, cheiro, olhares, toque, companheirismo, admiração, desejo, mas sim com o direcionamento reducionista para a relação penetrativa do ato sexual (Lima, Reis \& Demétrio, 2017).

\section{Eu tenho dificuldade de penetração durante a relação sexual. (H.21)}

Eu já não sou mais o mesmo homem, tenho muitas dificuldades nas atividades sexuais. (H.28)

Diante este olhar que reduz o conceito e o significado das sexualidades, torna-se um desafio fazer os homens perceberem que, embora possa haver alterações nos padrões sexuais, as expressões das sexualidades podem ser mantidas e ampliadas, sendo essa uma importante possibilidade para a minimização do desconforto, da ansiedade e dos sofrimentos gerados (Lima, Reis \& Demétrio, 2017).

Após o diagnóstico de câncer de próstata e o tratamento, o homem, de alguma forma, precisa compreender a necessidade de cuidar da sua saúde, como protagonista.

Hoje eu cuido bem melhor do que antes de ter os problemas que tive. Estou passando a me alimentar melhor, pois abusava de várias comidas pesadas. (H.4)

Hoje eu ainda caminho, mas não poderei mais andar de bicicleta. Eu vou sempre na consulta de 6 em 6 meses. Como frutas, legumes (H.6)

Me alimento bem, tenho uma alimentação balanceada, ainda não faço atividade física, durmo bem, não trabalho desde março devido a covid que fechou a escola onde eu trabalho. (H.9)

Diversos são os tratamentos disponíveis para o câncer de próstata e todos eles possuem efeitos colaterais. Um dos tratamentos mais utilizados para o câncer prostático, por exemplo, é a hormonioterapia, tendo em vista os resultados de eficácia que o tratamento tem mostrado na contenção do aumento do tumor. Contudo, este tratamento gera uma série de alterações hormonais no homem que causam efeitos colaterais como ginecomastia, ondas de calor, disfunção erétil, fadiga, dor óssea, entre outros. Essas alterações afetam o físico e o emocional desses homens, pois, além de provocarem alterações que modificam estruturas masculinas, geram sofrimento psicológico (Mota \& Barros, 2018).

Ferreira (2020) menciona que mudanças no estilo de vida repercutem no tratamento do câncer de próstata e melhoram a qualidade de vida do indivíduo, minimizam sintomas e diminuem a toxicidade do tratamento, entre outros pontos positivos. Ele afirma, ainda, que parte dos pacientes costumam ter uma boa aceitação nas mudanças alimentares, pois tem receio do que a doença pode acarretar e ressalta a existência de dificuldades na rotina de atividades físicas, devido às sequelas geradas pelo câncer.

Possuir uma rede de apoio familiar e profissional também reflete diretamente no trato da doença, pois adesão ao tratamento é um dos problemas encontrados pela equipe multiprofissional. Ter esse apoio facilita o paciente a enfrentar diversas situações que podem surgir, e que alteram o seu cotidiano e estilo de vida, além de fortalecer e incentivá-lo diante de uma condição que normalmente gera uma dificuldade de aceitação por parte dos pacientes (Rodrigues, Sá \& Zangrande, 2016). 


\section{Considerações Finais}

Ainda nos dias atuais, percebe-se um grande impasse voltado à saúde do homem, visto que estes se cuidam menos quando comparados à população feminina, que desde a infância é incentivada a identificar suas necessidades de saúde. Já os homens são vistos como fortes que não ficam doentes, aumentando sua vulnerabilidade para a doenças.

Existe uma multiplicidade de fatores de vulnerabilidade, individuais e sociais, que acabam comprometendo a promoção à saúde e à prevenção de doenças. O preconceito na realização de exames preventivos acaba dificultando o diagnóstico precoce, pois procuram as unidades de saúde apenas quando apresentam sintomas da doença, que, por vezes, já se encontra em estado avançado. Este fato diminui as chances de um tratamento efetivo, uma vez que dificulta atividades laborais e vida sexual ativa ou similar ao que era antes do diagnóstico.

Os homens do estudo não se percebem cuidando da sua saúde, de alguma forma ignorando as ações preventivas, como também desenvolvendo hábitos de vida não saudáveis. Esta situação reforça a necessidade, por parte das unidades básica de saúde e seus profissionais, de se desenvolver estratégias de conscientização e de captação dos homens a fim de que busquem a prevenção de doenças e comecem a cuidar de sua saúde.

Também foi apontado, pelos participantes do estudo, um declínio do desempenho sexual e/ou de sua abstenção, repercutindo na masculinidade e na autoestima. Isto só reforça o desempenho do papel social da masculinidade, afetando o psicológico e a vida do homem em seu cotidiano.

Cabe ressaltar que o estudo apresentou limitações próprias de uma investigação qualitativa, ao retratar a realidade de alguns homens com câncer de próstata, internados numa unidade urológica de um hospital universitário. Desta forma, os resultados não podem ser generalizados para a população masculina, apesar de os resultados condizerem com demais investigações nacionais.

O estudo traz contribuições para o âmbito da prevenção, ao entender que, através da disseminação do conhecimento, é possível estabelecer um diálogo que facilite a desconstrução do machismo, levando a população masculina a pensar em cuidar da sua saúde e, desse modo, prevenindo situações irreversíveis de agravo da doença.

Existem, ainda, lacunas que envolvem a saúde do homem. Nesse sentido, é imprescindível um acolhimento em sua integralidade na perspectiva de gênero, trazendo um desafio de se pensar a saúde do homem voltada à promoção à saúde e à prevenção de doenças. Assim, a educação em saúde revela-se um pilar para fortalecer o vínculo da população masculina com os serviços de saúde, de modo a dar-lhe a visibilidade necessária.

Além disso, o estudo contribui e incentiva novas pesquisas sobre a temática, bem como amplia o debate sobre gênero e suas implicações para o cuidado, principalmente quanto à saúde do homem, abrindo, assim, caminhos para a compreensão da evolução e aumento da visibilidade desta população.

\section{Referências}

Araújo, J. S., Conceição, V. M., Oliveira, R. A. A, \& Zago, M. M. F. (2015). Social and clinical characterization of men with prostate cancer treated at a university hospital. Revista Mineira de Enfermagem, 19(2), 196-203. 10.5935/1415-2762.20150035

Bardin, L. (2016). Análise de conteúdo. Tradução Luís Antero Reto, Augusto Pinheiro. Edições 70.

Brasil. Incidência de câncer no Brasil estimativa 2014. (2014). http://www.saude.sp.gov.br/resouces/ses/perfil/gestor/homepage/outros-destaque/estimativade-incidencia-de-cancer 2014/estimativa_cancer_24042014.pdf.

Brasil. Ministério da Saúde. (2008). Política Nacional de Atenção Integral à Saúde do Homem: Plano de Ação Nacional. Brasília. http://portalsaude.saude.gov.br/i ages/pdf/2014/maio/21/CNSH-DOC-Plano-Nacional.PNAISH-2009-2011.pdf.

Brasil. Ministério da Saúde. (2015). Câncer de próstata: causas, sintomas, tratamentos, diagnóstico e prevenção. Ministério da Saúde. de http://www.saude.gov.br/saude-de-a-z/cancer-de-prostata.

Ferrão, L., Bettinelli, L. A., \& Portella, M. R. (2017). Men's experiences with prostate cancer. Revista de Enfermagem UFPE online, 11(10), 4157-4164. 10.5205/reuol.10712-95194-3-SM.1110sup201720 
Ferreira, D. F. Uma análise do suporte e qualidade de vida após o diagnóstico e tratamento de câncer de próstata no setor de radioterapia. 2020. (Mestrado em Ciências) — Faculdade de Tecnologia Nuclear, Instituto de Pesquisas Energéticas e Nucleares. São Paulo. https://www.teses.usp.br/teses/disponiveis/85/85131/tde-17122020-111956/publico/2020FerreiraUmaAnalise.pdf.

Gomes, R. (2008). Sexualidade masculina, gênero e saúde. Fiocruz.

IBGE. (2010). Censo 2010 - Instituto Brasileiro de Geografia e Estatística. https://censo2010.ibge.gov.br.

Lima, C. F., Reis, A., \& Demétrio, F. (2017). Sexualidade e saúde: perspectivas para um cuidado ampliado. Bonecker Editora.

Lima, C. S. \& Aguiar, R. S. (2020). Men's access to primary health care services: an integrative review. Research, Society and Development, 9(4), e157943027. https://doi.org/10.33448/rsd-v9i4.3027

Lyra, J. A. et al. (2020). Men's know ledge about prostate cancer prevention in the Family health strategy. Research, Society and Development, 9(8), E07985346. https://doi.org/10.33448/rsd-v9i8.5346

Martins, E. R. C. et al. (2020b). Vulnerability of young men and their health needs. Esc. Anna Nery, 24(1), e20190203. 10.1590/2177-9465-EAN-2019-0203

Martins, E. R. C. et al. (2020a). Young men's health from the perspective of health promotion and disease prevention. Brazilian Journal of Health. 3(2), 23012308. 10.34119/bjhrv3n2-081

Martins, E. R. C. et al. (2020c). Young men's health from the perspective of health promotion and disease prevention. Research, Society and Development, 9(9), e400997351. 10.33448/rsd-v9i9.7351

Minayo, M. C. S. (2013). O desafio do conhecimento: pesquisa qualitativa em saúde. Hucitec.

Mota, T. R., \& Barros, D. P. O. (2018). Perfil dos pacientes com câncer de próstata em hospital de referência no estado de Pernambuco. Revista Brasileira de Análises Clínicas, 50(4), 334-338. 10.21877/2448-3877.201900766

Rego, R. F. N. B. et al. (2020). Epidemiological clinical profile of the population served in a prostate cancer screening program. Revista de Atenção à Saúde, 18(65), 38-47. https//doi.org/10.13037/ras.vol18n65. 664

Rodrigues, R. S., Sá, R. G., \& Zangrande, C. (2016). Epidemiological profile of patients with prostate cancer treated at there ferralcenter for oncology in the city of Sinop /MT. Enfermagem Brasil, 15(4), 181-189. https://doi.org/10.33233/eb.v15i4.497

Silva, M. H. A., Costa, W. S., Sampaio, F. J. B., \& Souza, D.B. (2018). The corpus cavernosum after treatment with dutasteride or finasteride: A histomorphometric study in a benign prostatic hyperplasia rodent model. Asian J Androl. 20(5), 505-510. 10.4103/aja.aja_28_18 